\title{
Valtorcitabine Dihydrochloride
}

National Cancer Institute

\section{Source}

National Cancer Institute. Valtorcitabine Dihydrochloride. NCI Thesaurus. Code C90788.

A dihydrochloride salt form of valtorcitabine, a prodrug form of torcitabine (Ldeoxycytidine), a nucleoside analog with activity against hepatitis B virus. Torcitabine is modified in vivo to its triphosphate form and acts as a competitive inhibitor of viral DNA polymerase. 